This item was submitted to Loughborough's Research Repository by the author.

Items in Figshare are protected by copyright, with all rights reserved, unless otherwise indicated.

\title{
The extended growth of graphene oxide flakes using ethanol CVD
}

PLEASE CITE THE PUBLISHED VERSION

http://dx.doi.org/10.1039/c3nr33704a

PUBLISHER

RSC Publishing (journal (@ The Royal Society of Chemistry; article @ the authors)

VERSION

SMUR (Submitted Manuscript Under Review)

LICENCE

CC BY-NC-ND 4.0

REPOSITORY RECORD

Huang, Jingfeng, Melanie Larisika, Derrick W.H. Fam, Qiyuan He, Myra A. Nimmo, Christoph Nowak, and Alfred I.Y. Tok. 2019. "The Extended Growth of Graphene Oxide Flakes Using Ethanol CVD”. figshare. https://hdl.handle.net/2134/12940. 
This item was submitted to Loughborough's Institutional Repository (https://dspace.lboro.ac.uk/) by the author and is made available under the following Creative Commons Licence conditions.

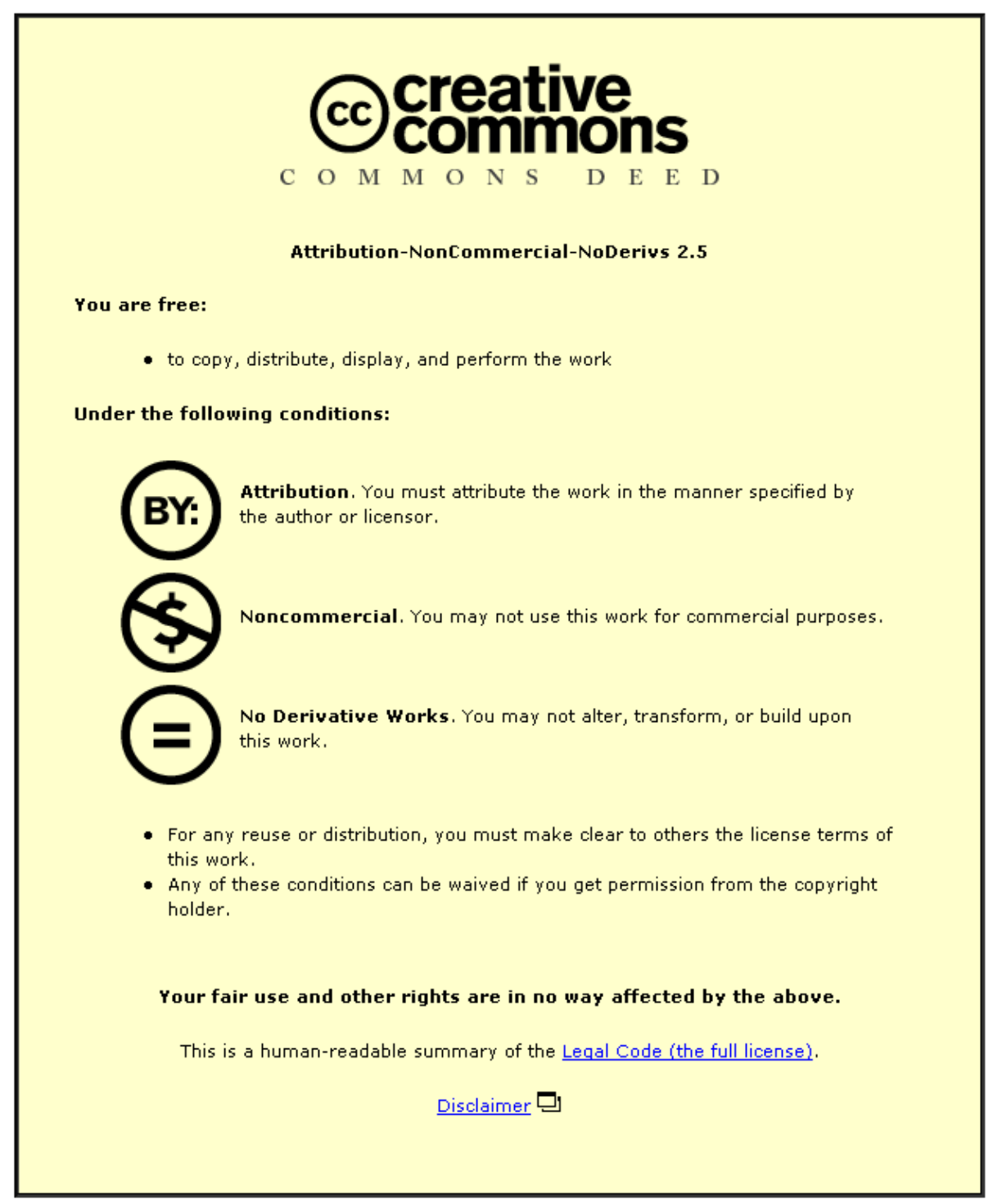

For the full text of this licence, please go to: http://creativecommons.org/licenses/by-nc-nd/2.5/ 


\title{
The Extended Growth of Graphene Oxide Flakes Using Ethanol CVD
}

\author{
Jingfeng Huang, ${ }^{\text {a,b }}$ Melanie Larisika ${ }^{\mathrm{c}, \mathrm{d}}$ Derrick Fam W. H., ${ }^{\mathrm{a}}$ Qiyuan He, ${ }^{\mathrm{a}}$ Myra A. Nimmo, ${ }^{\mathrm{e}}$ Christoph \\ Nowak $^{*, \mathrm{~d}}$ and Alfred Tok I. Y., ${ }^{*, \mathrm{~b}}$
}

\author{
5 Received (in $X X X, X X X$ ) Xth $X X X X X X X X X 20 X X$, Accepted Xth $X X X X X X X X X 20 X X$ \\ DOI: $10.1039 / b 000000 x$
}

We report the extended growth of Graphene Oxide (GO) flakes using atmospheric pressure ethanol Chemical Vapor Deposition (CVD). GO was used to catalyze the deposition of carbon on substrate in the ethanol CVD with $\mathrm{Ar}$ and $\mathrm{H}_{2}$ as carrier gases. Raman, SEM, XPS 10 and AFM characterized the growth to be reduced GO (RGO) of < 5 layers. This new grown RGO possesses lower defect density with larger and increased distribution of $\mathrm{sp}^{2}$ domains than chemically-reduced RGO. Furthermore this method without optimization reduces relative standard deviation of electrical conductivity between chips, from $80.5 \%$ to $16.5 \%$, enabling RGO to be used in practical electronic devices.

\section{1. Introduction}

Graphene is a single atomic layer of $\mathrm{sp}^{2}$ hybridized carbon atoms arranged in a honey-comb structure. Since the exfoliation of graphite into individual graphene sheets in $2004,{ }^{1}$ its large carrier mobility, extraordinary thermal, chemical and optical properties 20 have been highlighted. ${ }^{2}$ However the semi-metal and zero-band gap electronic structure of pristine graphene limits its use in electronic, sensing and optical applications. Some approaches to open the band-gap include post-processing of graphene such as strain engineering, ${ }^{3}$ lateral confinement, ${ }^{4}$ breaking inversion ${ }_{25}$ symmetry in bi-layer graphene, ${ }^{5}$ oxidation ${ }^{6}$ and usage of reduced graphene oxide (RGO). ${ }^{7,8}$

One method of obtaining a band gap is through the use of RGO. RGO is an inexpensive, aqueous processable material with an industrial-scalable production route. An efficient and scalable 30 method for production of RGO involves chemical oxidation of graphite followed by exfoliation and reduction. ${ }^{9}$ After reduction, the increased pockets of $\mathrm{sp}^{2}$ hybridised carbon structure provides its mechanical strength and electrical conductivity. ${ }^{7}$

Despite these advantages and a substantial progress in RGO 35 research over the years, we are still not able to produce RGO on substrates with good homogeneity and conductivity for practical applications. ${ }^{10}$ This is due to the incomplete, poor coverage and different sizes of RGO flakes on substrates; posing a challenge to working with RGO fabrication.

40 It has been reported that Carbon Nanotubes (CNT) can be grown in ethanol Chemical Vapor Deposition (CVD) furnace using metal catalysts. But recently, it has been discovered that CNT can also be grown without a metal catalyst. A convincing model is still lacking on how carbon is nucleated and grown from 45 oxides. Carbon growth has been reported using oxide seeds, such as nano-diamond particles, ${ }^{11} \quad \mathrm{ZrO}^{12}$ and $\mathrm{SiO}_{\mathrm{x}}{ }^{13}$ via vapor-solid-solid (VSS) growth mechanisms. Attempts have been made to grow Graphene on substrates directly without any catalyst ${ }^{14}$ using plasma or high pressure. Attempts have also been 50 made to repair Graphene and Graphene oxide using ethanol $\mathrm{CVD}^{15,16}$ but no growth was observed.

Metals are commonly used as catalysts in several types of chemical reactions such as carbon nano-tube growth ${ }^{17}$ and Haber-Weiss reaction. ${ }^{18}$ One of the main properties of those 55 metals is the presence of a free electron cloud on the surface of the metal which allows for ready exchange between the metal surface and the electron acceptor molecules. This is the principle behind catalytic reactions the metal acts as electron donor or in some cases as an electron acceptor. From graphene it is known 60 that it provides $2 \mathrm{D}$ electron gas properties, comparable with metal surfaces. ${ }^{19}$

From existing literature it is known that carbon cannot crystallize well by deposition from carburizing gases at low temperature without catalytic activation because of its strong ${ }_{65} \mathrm{C}-\mathrm{C}$ bonds and high melting temperature. To form good crystalline carbon, the carbon atoms must dissolve, diffuse through metal particles, and crystallize on an appropriate facet that can act as a template to help the epitaxial growth of carbon crystals. ${ }^{20}$ In our experiment the RGO on the substrate can act 70 similar to the described metal particles, and help the growth of carbon crystals/layers. Here, we report the growth of RGO, which not only extend size but also homogeneity of its electrical conductivity, enabling RGO in electronic devices by the extended growth of GO flakes using ethanol CVD.

\section{2. Results and Discussion}

To grow and extend the size of RGO, a modified Hummers ${ }^{10,21}$ method was first used to obtain Graphene Oxide (GO) sheets. Briefly, graphite flakes were pre-oxidized using concentrated $\mathrm{H}_{2} \mathrm{SO}_{4}$, ultra-sonicated and filtered to remove small graphite 80 debris. Then, the dried pre-oxidized graphite powder was added 


\author{
Cite this: DOI: $10.1039 / \mathrm{c} 3 n r 33704 a$
}

\title{
pubs.rsc.org/en/content/articlelanding/2013/NR/c3nr33704a
}

\section{Before Growth}

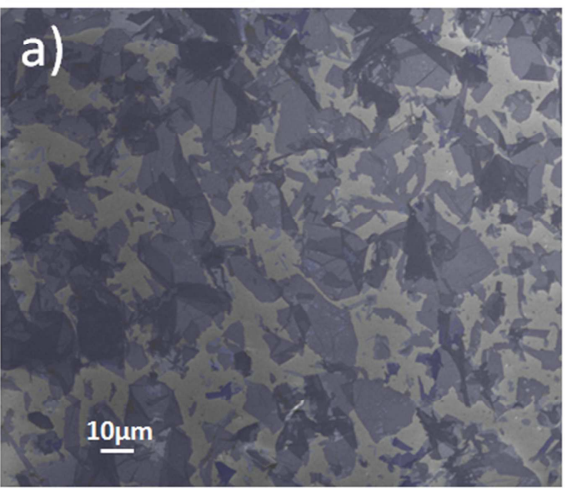

\section{After Growth}

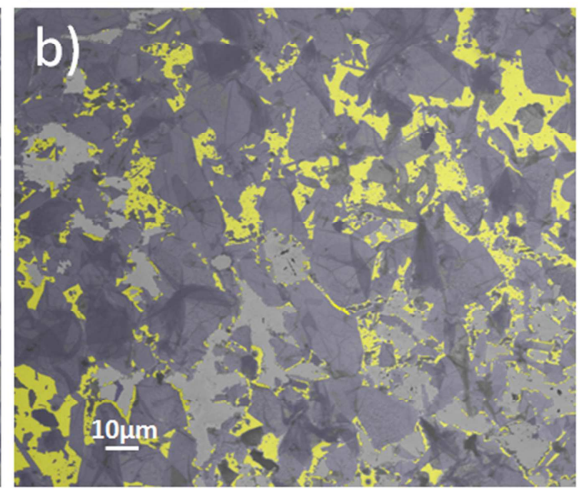

High Magnification

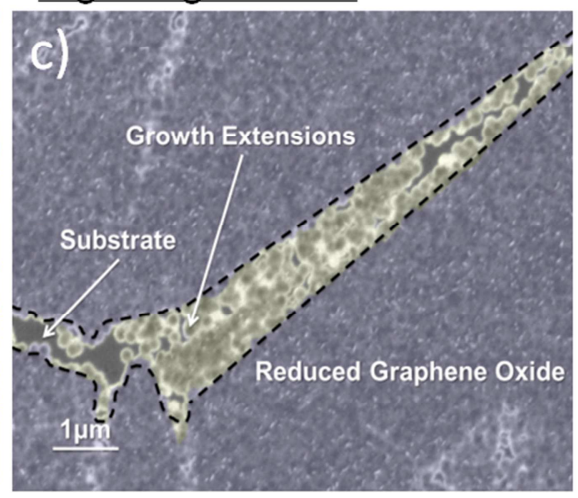

Fig. 1 SEM image of a) before ethanol CVD treatment GO flakes [highlighted in purple] on $\mathrm{SiO}_{2}$ substrate with ca. 60\% coverage b) after a 30-minutes CVD treatment RGO flakes [highlighted purple] and the extended RGO growths [highlighted in yellow] with a total $c a$. 75\% coverage. c) Highmagnification image on the new growths bridging between two RGO flakes.
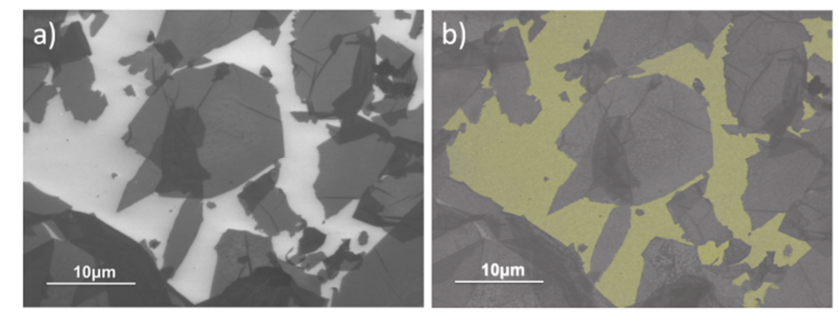

Fig. 2 SEM image of a) GO flakes before and b) RGO flakes and extended RGO growths [highlighted in yellow] after 2 hours ethanol

CVD treatment. The size and features of the Graphene Oxide are preserved after the CVD treatment.

10 into concentrated $\mathrm{H}_{2} \mathrm{SO}_{4}$ and $\mathrm{KMnO}_{4}$. Thereafter, the $\mathrm{GO}$ was electrostatically attached to the APTES-functionalized $\mathrm{SiO}_{2}$ substrates.

The substrates, with 1-2 layers of GO on top, were placed on a holder and placed in a CVD tube furnace setup (Figure S1). The 15 furnace was purged with Ar for 30 minutes at $300 \mathrm{sccm}$ to remove $\mathrm{O}_{2}$ that can react with the $\mathrm{GO}$ to form $\mathrm{CO}$ and $\mathrm{CO}_{2}$. After purging, the flow of Ar was adjusted to $100 \mathrm{sccm}$ and $\mathrm{H}_{2}$ turned on to $20 \mathrm{sccm}$. Next, the valves to ethanol precursor were turned on and the furnace heated to $950^{\circ} \mathrm{C}$ at $40^{\circ} \mathrm{C}$ per minute. The gases 20 were passed through an ethanol bubbler held at $0^{\circ} \mathrm{C}$ in a water bath chiller before entry into the furnace. The rate of ethanol consumed was $2.5 \mathrm{ml} /$ hour. After 2 hours, the furnace was turned off and the tube left to cool slowly to room temperature while $\mathrm{H}_{2} /$ Ar continues to flow.

25 A typical SEM image of $\mathrm{GO}$ flakes on $\mathrm{SiO}_{2}$ substrate is shown in Figure 1a. After a 30-minutes ethanol CVD treatment, the total RGO coverage increased to ca. $75 \%$ (yellow highlighted area) shown in Figure 1b. Figure 1c shows a high-magnification SEM image on the new growths bridging between edges of two ${ }_{30}$ RGO flakes.
A TEM image of the boundaries between the existing flake and new growth after 2 hours ethanol CVD treatment is shown in Figure S2. In the TEM image, the lighter contrast of the newly grown RGO is highlighted in yellow. The contrast difference is 35 due to the difference in number of layers between the pre-existing RGO flake and newly grown RGO. It is observed that the new growth ends with circular edges, similar to observations in the high-magnification SEM image.

To clarify the effect of the GO, we have subjected clean 40 substrates and also APTES-functionalized substrates to the same ethanol CVD process for up to 2 hours. These control substrates without initial GO flakes, showed neither carbon deposit nor carbon spectrum on the SEM and Raman respectively. This shows that initial GO flakes are essential to enable further RGO 45 growth in the ethanol CVD setup. Obviously the initial RGO flakes seem to act as a crystal nucleus for further growth of these flakes.

Observation from the SEM picture of the same GO flakes before and after CVD treatment (Figure 2a and 2b) shows that 50 the original GO is still present after the CVD treatment and there is no change in size. However, a different contrast between the original graphene flake and substrate can be observed under the SEM. Also, the extended growths are observed to be lighter in shade compared to pre-existing flakes. A possible contrast 55 mechanism could be e- beam-induced surface potential between graphene of different band gaps. ${ }^{18}$ Differences in the number of RGO layers can also be observed as different contrast in the picture, the lightest being single or few layers and darker indicating more layers. Thus using the SEM allows effective and 60 efficient identification of the existing flake, the new growth area and the relative thickness of the RGO. Within 2 hours, the substrate of $c a .1 \mathrm{~cm} \times 1 \mathrm{~cm}$ is completely covered with RGO. 


\section{Cite this: DOI: 10.1039/c3nr33704a}

\section{pubs.rsc.org/en/content/articlelanding/2013/NR/c3nr33704a}

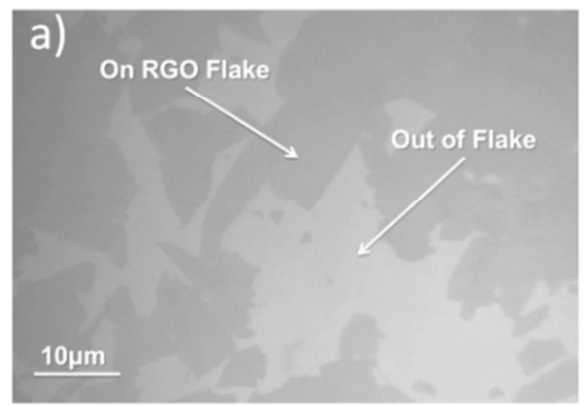

b)

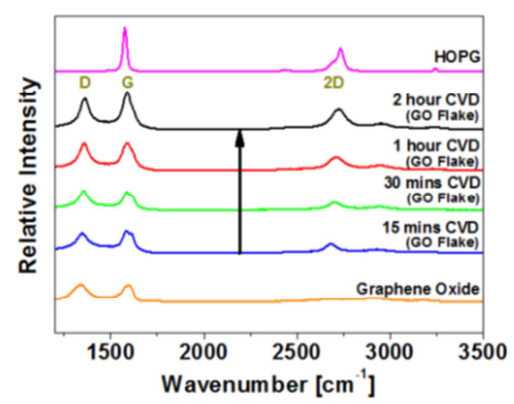

c)

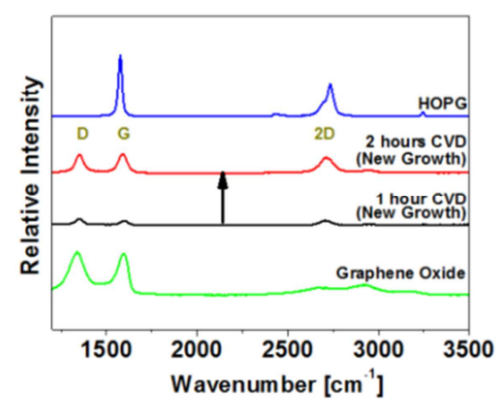

Fig. 3 a) Optical micrograph [100x magnifications] of RGO flakes treated with 2-hours of ethanol CVD, showing contrast difference between RGO flake and out of flake areas. Raman spectra [488nm] on b) RGO flake and c) out of flake areas, with different CVD processing times, in comparison to GO and Highly Ordered Pyrolytic Graphite (HOPG).
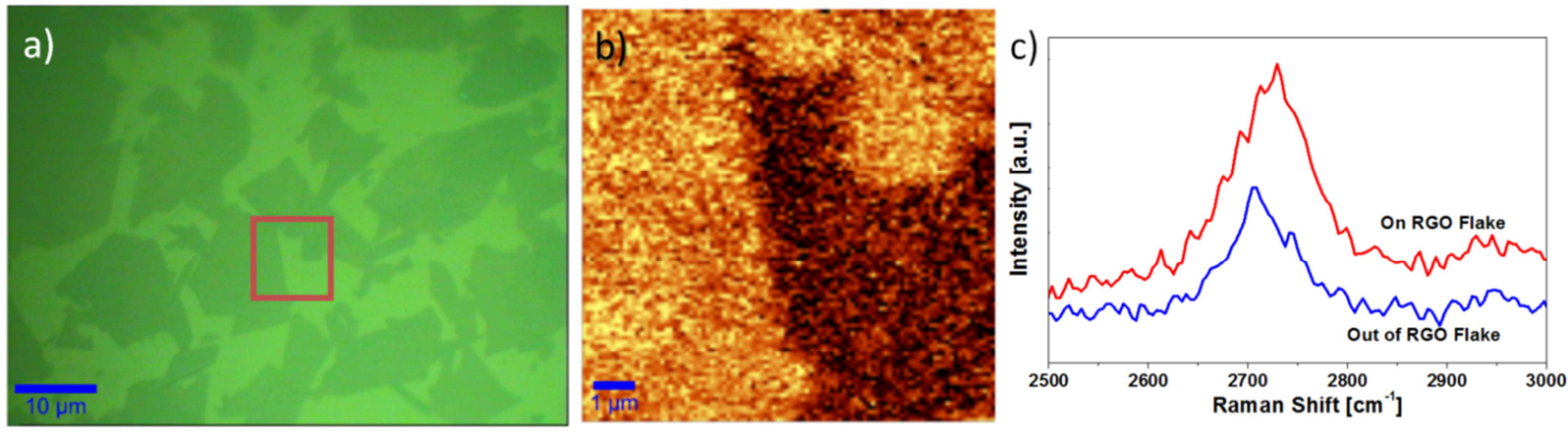

Fig. 4 a) Optical image of 2-hour ethanol CVD treated $\mathrm{RGO}$ on $\mathrm{SiO}_{2}$ b) Raman map $\left(10 \mathrm{x} 10 \mu \mathrm{m}^{2}\right)$ constructed based on difference in $2 \mathrm{D}$ peak position. Increasing layers up-shifts the 2D peak position [brighter shade represents peaks that are more up-shifted]. c) 2D-band Raman [488nm] spectrum of pre-existing RGO flake and spaces between flakes used to construct the Raman map.

10 Raman spectroscopy is important for the characterization of the graphene structure, thickness and defects. The optical micrograph of RGO flake and the substrate is shown in Figure 3a. Figure 3b shows Raman spectrum on the RGO flakes processed at different CVD durations. Both Graphitic-band (G15 band, $\sim 1575 \mathrm{~cm}^{-1}$ ) and second-order Defect-band (2D-band, $\sim 2700 \mathrm{~cm}^{-1}$ ) are observed in Highly Ordered Pyrolytic Graphene (HOPG), GO and RGO in agreement with the literature. ${ }^{23}$ The Gband of unprocessed GO $\left(1599 \mathrm{~cm}^{-1}\right)$ was up-shifted compared with that of HOPG $\left(1575 \mathrm{~cm}^{-1}\right)$ and can be attributed to the 20 presence of isolated double bonds that resonate at a higher frequency in GO than graphite. ${ }^{24}$ The G-band of the 2 hour CVD processed sample $\left(1585 \mathrm{~cm}^{-1}\right)$ down-shifts closer to the G-band of HOPG. Furthermore, the 2D-band up-shifting of CVD samples towards the graphite 2-D band position also suggests more 25 significant structural changes towards graphite as CVD process time increases. $^{25}$

The observation of the D-band of GO and RGO at $\sim 1350 \mathrm{~cm}^{-1}$ suggests the presence of disordered structural defects from the attachment of hydroxyl and epoxide groups on the carbon plane. ${ }_{30}{ }^{23}$ The Defect-band/Graphitic-band (D/G) intensity ratio is observed to decrease from 1.02 in GO samples to 0.88 in the 2 hour CVD samples. Decrease in the D/G intensity value corresponds to an increase in average size of $\mathrm{sp}^{2}$ domains. ${ }^{26}$ Thus, ethanol CVD method increased the average size of $\mathrm{sp}^{2}$ domains 35 on existing GO flakes. Using the Tuinstra-Koenig ${ }^{27}$ empirical method, the lateral domain size of the $\mathrm{sp}^{2}$ graphitic ring cluster on the GO flake after 2 hours of Ethanol CVD treatment is calculated to be $\sim 4.8 \mathrm{~nm}$. The treatment by ethanol CVD could increase the spatial dimension of the $\mathrm{sp}^{2}$ region of existing RGO 40 flake thus resulting in an increase in conductivity. However, it is observed that this graphitization is not the only reason that led to the increase in electrical conductivity.

Raman spectroscopy was also performed on the spaces between RGO flakes for all samples as shown in Figure 3c. The 45 contrast difference between the initial RGO flakes and the substrate was used as a guide to perform Raman. Measurement of spaces from the 1 hour and 2 hours samples showed peaks of $1351 \mathrm{~cm}^{-1}, 1585 \mathrm{~cm}^{-1}, 2711 \mathrm{~cm}^{-1}$ which are consistent with the D, G and 2D bands of typical RGO. Whereas, no carbon Raman 50 signals were obtained for the 15 minutes and 30 minutes CVD processed chips, consistent with our SEM observation 
Cite this: DOI: 10.1039/c3nr33704a

pubs.rsc.org/en/content/articlelanding/2013/NR/c3nr33704a

a)

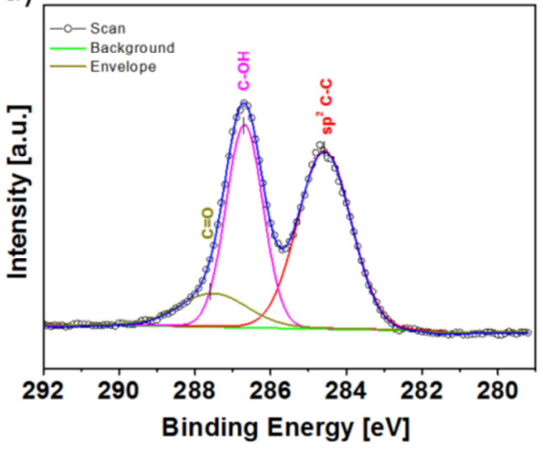

b)

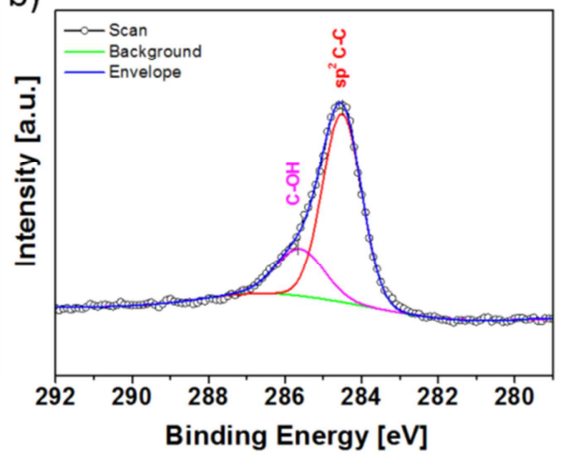

c)

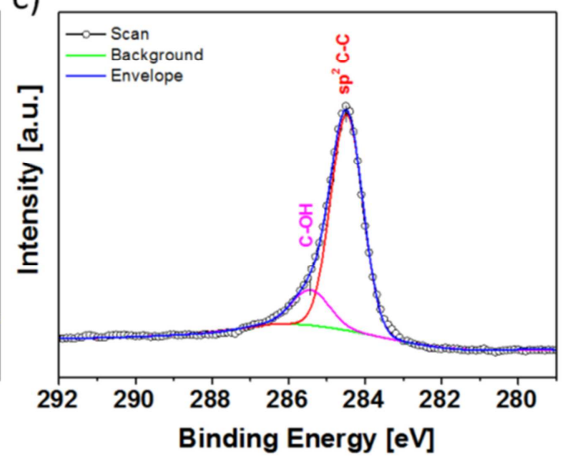

Fig. 5 XPS spectra and identified binding energies for a) GO and b) RGO after 15 mins and c) 120 mins of ethanol CVD treatment.

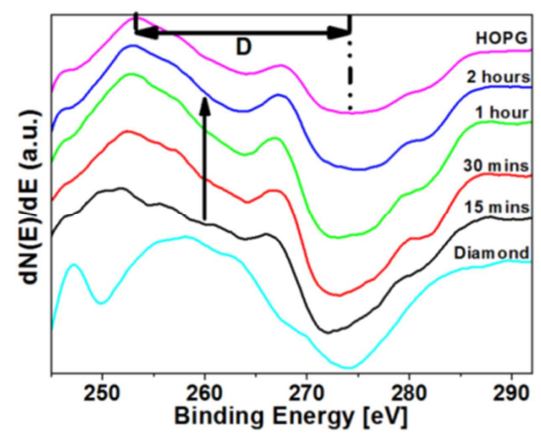

Fig. 6 First derivative of the X-ray excited C KLL spectra of HOPG, CVD treated samples and diamond. The $\mathrm{D}$ value which corresponds to the area ratio of $\mathrm{sp}^{2}$ increases with increasing ethanol CVD process time.

that RGO growth starts from the edges and does not fill up the large spaces in the 15 and 30 minutes samples. The Raman G10 band carbon intensity of the 1 hour sample and GO flake was $\sim 70 \%$ and $\sim 40 \%$ of the 2 hour sample's intensity respectively. Since Raman intensity of the allowed peak is proportional to the volume of sample, ${ }^{28}$ the intensity difference suggests a smaller amount of new RGO in the 1 hour versus the 2 hour sample.

15 The G-band and the 2-D band of the RGO growths are also observed to down and up-shift respectively as CVD time increases. Both bands shift towards that of HOPG, an indication of structural changes towards graphite as process time increases.

${ }^{21}$ The D/G peak intensity ratio of the RGO growth in the 2 hours

${ }_{20}$ CVD sample decreases to 0.71 from GO's ratio of 1.02 , suggests a significant increase in the average size of the $\mathrm{sp}^{2}$ domain. This new RGO growth's D/G intensity ratio of 0.71 is remarkably low compared to 0.88 on RGO flakes after 2 hours ethanol CVD or 1.56 on RGO flakes reduced with 12 hours of hydrazine vapor. ${ }^{17}$

${ }_{25}$ Using the Tuinstra-Koenig empirical formula, ${ }^{27}$ the lateral domain size of the $\mathrm{sp}^{2}$ graphitic ring cluster on the newly grown $\mathrm{RGO}$ is found to be $\sim 5.7 \mathrm{~nm}$.

In the 2D-band of HOPG and in the graphene sheets of 5 or more layers, there are 2 components, $2 \mathrm{D}_{1}\left(2690 \mathrm{~cm}^{-1}\right)$ and $2 \mathrm{D}_{2}$ $30\left(2732 \mathrm{~cm}^{-1}\right)$ as reported earlier. ${ }^{23}$ Figure $\mathbf{S 3}$ shows this deconvoluted $2 \mathrm{D}_{1}$ and $2 \mathrm{D}_{2}$ bands of HOPG. It is noted that in our ethanol CVD treated chips, both new growth and pre-existing GO flakes, do not show the $2 \mathrm{D}_{1}$ and $2 \mathrm{D}_{2}$ bands typical of HOPG, thus suggesting the thickness of the layers must be less than 5 layers, 35 which we confirm with the Atomic Force Microscopy (AFM) later in this manuscript.

Raman spectroscopy can distinguish the number of layers of carbon. Increasing the number of layers up-shifts the Raman 2D peak position. ${ }^{23,29}$ A Raman image can then be reconstructed by 40 mapping out the peak position in our samples. This reconstruction is shown in Figure 4a and $4 \mathbf{b}$. The peak position shift of $23 \mathrm{~cm}^{-1}$ between the $2 \mathrm{D}$ peaks is shown in Figure 4c. From the Raman map, the overall coverage of the substrate by pre-existing RGO and newly grown RGO is $c a$. $100 \%$.

$45 \quad$ Figure 5 reports the C1s XPS spectra of original GO, 15 mins CVD-processed RGO and 120 mins CVD-processed RGO. In Figure 5a, the $\mathrm{C} 1 \mathrm{~s}$ signal can be deconvoluted into $\mathrm{C}-\mathrm{C}$ (284.6eV), C-OH (286.3eV) and $\mathrm{C}=\mathrm{O}$ (287.2). These assignments are in agreement to previous reports. ${ }^{10,25}$ After CVD treatment of 5015 minutes (Figure 5b), the carbonyl and epoxy acid signatures were diminished from their starting intensities and were dwarfed by the C-C signals. The atomic percentage (at \%) for the different functional groups are calculated with respect to the total area of the $\mathrm{C} 1 \mathrm{~s}$ peak. C-C bond accounted for 49.6, 76.5 and 84.2 at $\%$ in 55 the RGO sample after CVD treatment of 0,15 and 120 mins respectively. The $\mathrm{C}=\mathrm{O}$ bond peak (initially at 9.7 at\%) was completely removed after 15 mins of CVD treatment and could possibly be reduced to $\mathrm{C}-\mathrm{O}$ bonds, a reduced species arising from reduction of the $\mathrm{C}=\mathrm{O}$ in $\mathrm{GO}$. Between the 15 and 120 mins 60 sample the C-O at\% decreased from 23.5 to 15.8 at\%, suggesting that graphitization and growth has taken place. The XPS results confirms the identity of reduced graphene oxide, complete removal of $\mathrm{C}=\mathrm{O}$ bonds and that graphitization and growth can continue up to 120 mins as shown in Figure 5c. 


\author{
Cite this: DOI: 10.1039/c3nr33704a
}

\title{
pubs.rsc.org/en/content/articlelanding/2013/NR/c3nr33704a
}
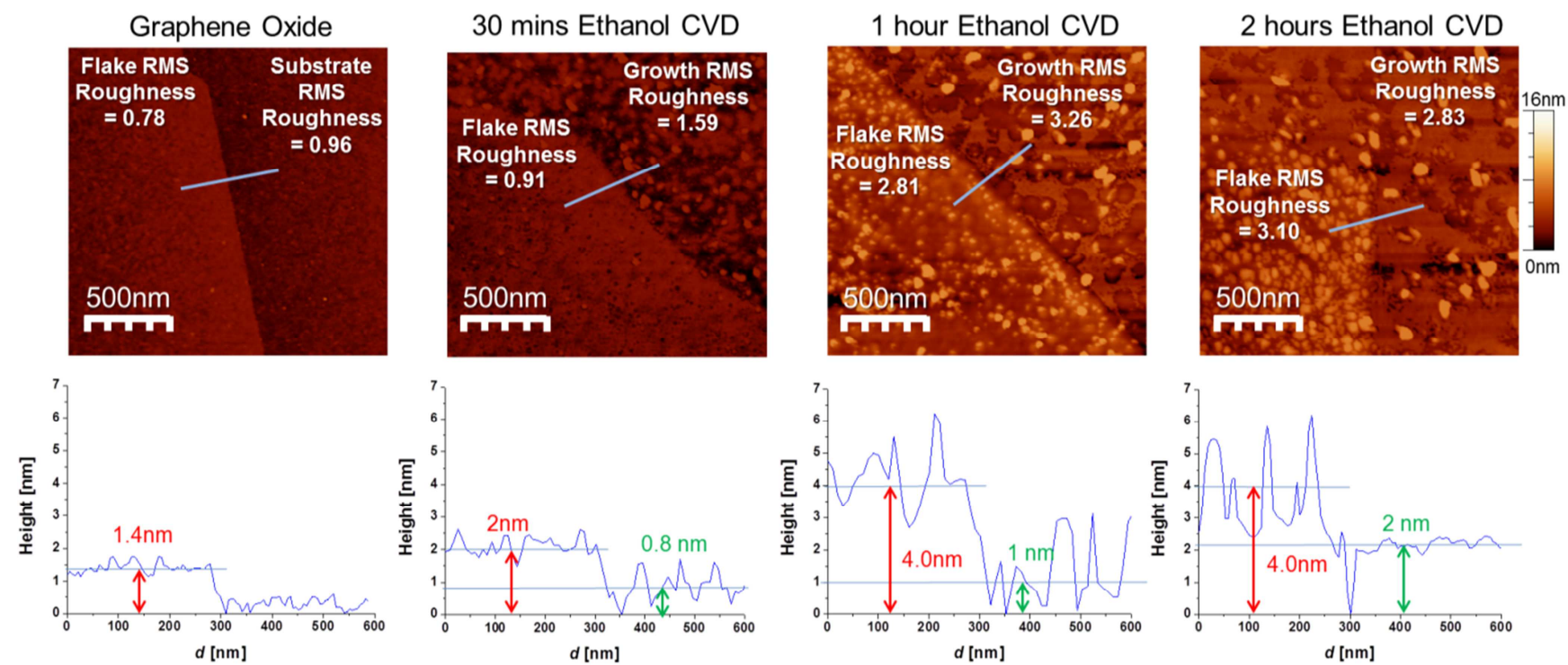

Fig. 7 AFM images with Root Mean Squared (RMS) roughness value and corresponding height profile of Graphene oxide with different stages of CVD process.

Table 1. D parameter and $\mathrm{sp}^{2}$ percentage area of different carbon samples

\begin{tabular}{ccc}
\hline Samples & $\mathrm{D}(\mathrm{eV})$ & $\% \mathrm{sp}^{2}$ \\
\hline HOPG & 21.87 & 100 \\
RGO (2 hours CVD) & 21.45 & 93 \\
RGO (1 hour CVD) & 20.00 & 77 \\
RGO (30 mins CVD) & 19.79 & 66 \\
RGO (15 mins CVD) & 19.58 & 63 \\
RGO ( 12 hours hydrazine vapor) & 18.74 & 50 \\
GO & 18.23 & 42 \\
\hline
\end{tabular}

In addition to $\mathrm{C} 1 \mathrm{~s}$ spectra, the $\mathrm{C}$ KLL spectra were also measured to compare $\mathrm{sp}^{2}$ to $\mathrm{sp}^{3}$ ratio in the treated samples. Figure 6 shows the spectra of the first derivative of X-ray excited C KLL between diamond, HOPG and CVD treated samples 10 (treatment times $=15,30,60,120$ minutes). The $\mathrm{D}$ value between the maximum of the positive-going excursion and the minimum of the negative-going excursion in the derivative XAES spectra represents the energy separation. This D value conforms to linear proportionality between the graphite and diamond. ${ }^{30}$ Thus 15 increasing D is associated to the increasing number of $\pi$ electrons and the percentage of $\mathrm{sp}^{2}$ area in the film. ${ }^{31,32}$ The assignment of peaks for $\mathrm{D}$ value calculations are as reported in previous literature. $^{33}$

The $\mathrm{D}$ values and calculated $\mathrm{sp}^{2}$ percentage through linear 20 interpolation between HOPG and diamond are tabulated in Table 1. With increasing treatment time, the percentage of $\mathrm{sp}^{2}$ increases, due to the contributions from graphitization of existing flakes and new enlarged growth of the RGO. The calculated $42 \% \mathrm{sp}^{2}$ area in as-synthesized GO is in good agreement with literature. ${ }^{7}$ The 25 percentages of $\mathrm{sp}^{2}$ sites on RGO increased to $93 \%$ with 2 hours of ethanol CVD treatment. This $\mathrm{sp}^{2}$ sites increase corresponds to a factor of more than 2 times from the initial GO flake.

AFM images with RMS roughness value and corresponding 30 height profile of RGO flake at different ethanol CVD treatment time are shown in Figure 7. In the first 30 minutes of ethanol CVD treatment, the thickness of graphene oxide flake increases to $\sim 2 \mathrm{~nm}$; after which carbon islets of $\sim 2 \mathrm{~nm}$ start to form on the GO flake and increase in distribution. These islets on the flake do 35 not agglomerate into a continuous layer. This is reflected in the RMS roughness of the RGO flake which increases significantly from 0.9 (30 mins ethanol CVD treatment) to 3.1 (2 hour ethanol CVD treatment). This 2-4nm height corresponds to 2-4 layer of GO flakes ${ }^{34}$ and is in agreement with our Raman observation of 40 less than 5 RGO layers.

After 30 minutes of ethanol CVD treatment, carbon islets of diameter $<50 \mathrm{~nm}$ are observed outside the RGO flake. In contrast, to the islets formed on RGO flakes, the islets on the substrate agglomerate as time progresses, to larger islets of $\sim 80 \mathrm{~nm}$ in the 1 45 hour sample and finally into a layer up to $400 \mathrm{~nm}$ across in the 2 hour sample. This agglomeration corresponds to a peak in RMS value at 3.3 (1 hour CVD) which then decreases to 2.8 (2 hours CVD).

We also investigated the effect of CVD process time on the 50 electrical resistance on the samples using a 4-point probe setup. Samples were reduced differently with 15, 30, 60 and 120 minutes of ethanol CVD and hydrazine vapor. 5 samples were prepared for each condition. Their electrical resistance and standard deviation were then measured, as shown in Table 2. For 55 accurate comparison, all the chips were made in one single batch. The average resistance of samples after 15 minutes of ethanol 
Cite this: DOI: 10.1039/c3nr33704a

pubs.rsc.org/en/content/articlelanding/2013/NR/c3nr33704a

Table 2. Electrical measurements of RGO processed under different conditions

\begin{tabular}{|c|c|c|c|c|c|}
\hline \multirow[t]{2}{*}{ Reduction Conditions } & \multirow{2}{*}{$\begin{array}{l}\text { Hydrazine Vapor Reduction } \\
12 \text { hours } \\
\end{array}$} & \multicolumn{4}{|c|}{ Ethanol CVD Reduction } \\
\hline & & $15 \mathrm{mins}$ & $30 \mathrm{mins}$ & 1 hour & 2 hours \\
\hline $\begin{array}{l}\text { Average Resistance } \\
(\Omega / \square)\end{array}$ & $1.99 \mathrm{E}+06$ & $6.10 \mathrm{E}+04$ & $2.87 \mathrm{E}+04$ & $2.23 \mathrm{E}+04$ & $4.68 \mathrm{E}+03$ \\
\hline $\begin{array}{l}\text { Absolute Standard } \\
\text { Deviation }(\Omega)\end{array}$ & $1.60 \mathrm{E}+06$ & $4.42 \mathrm{E}+04$ & $1.25 \mathrm{E}+04$ & $7.14 \mathrm{E}+03$ & $7.70 \mathrm{E}+02$ \\
\hline $\begin{array}{l}\text { Relative Standard } \\
\text { Deviation }(\%)\end{array}$ & 80.5 & 72.6 & 43.6 & 32.1 & 16.5 \\
\hline
\end{tabular}

CVD is 2 magnitudes less than hydrazine reduced samples. This could be due to the improved graphitization of the RGO as shown in our Raman and XPS analysis. However, the relative standard 5 deviation for these 15 mins treated sample only decreased marginally by $9.8 \%$. We highlight that as CVD treatment time increases to 2 hours; the electrical resistance decreased by $99.8 \%$ and the relative standard deviation decreased by $79.5 \%$ compared to hydrazine vapor reduced samples. This resistance drop can be 10 attributed to improvement between inter-flake connections while the standard deviation drop is attributed to the complete $100 \%$ RGO coverage of sample chips, as show in SEM and Raman Mapping.
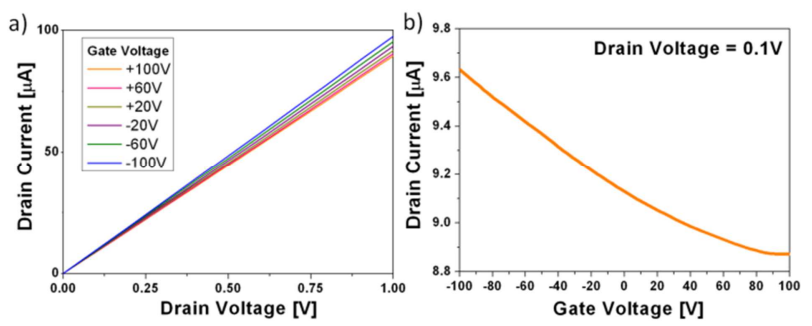

15 Fig. 8 Output characteristics $I_{d}-V_{d}$ (drain current- drain voltage) of the transistor device under different applied gate voltages and Id-Vg (drain current-gate voltage) transfer curve of bottom-gated graphene FET under sweeping $\mathrm{V}_{\mathrm{g}}$.

Back-gated Field-Effect Transistors (FET) based on 2-hour ${ }_{20}$ ethanol CVD processed RGO sheets was fabricated and their electrical properties tested under ambience temperature and pressure. The channel length of $100 \mu \mathrm{m}$ was chosen to ensure that the transport is bulk limited and the role of contacts minimized.

Figure 8a illustrates a typical drain current $\left(\mathrm{I}_{d}\right)$ versus drain 25 voltage $\left(\mathrm{V}_{\mathrm{d}}\right)$ curve prepared with ethanol CVD processed RGO field-effect transistor at six discreet gate voltages $\left(\mathrm{V}_{\mathrm{g}}\right)$. The linear output behaviour indicated good ohmic contact between the RGO film and electrodes. Figure $8 \mathbf{b}$ illustrates the same transistor $I_{d}$ under sweeping $\mathrm{V}_{\mathrm{g}}$ at fixed $\mathrm{V}_{\mathrm{d}}=0.1 \mathrm{~V}$. The charge neutrality point 30 is shifted to the positive side due to p-doing from persistent moisture and oxygen. ${ }^{35}$

The hole mobility is then extracted from the linear regime of the transfer characteristics using the equation $\mu=(L /$ $\left.W C_{o x} V_{d}\right)\left(\Delta I_{d} / \Delta V_{g}\right)$ where $\mathrm{L}$ and $\mathrm{W}$ are the channel length and 35 width, Cox the gate capacitance (which is $1.15 \times 10^{-9} \mathrm{~F} / \mathrm{cm}^{2}$ for a gate oxide of $300 \mathrm{~nm}$ ), Vd, Id, Vg are drain-source voltage, drainsource current and gate voltage, respectively. The mobility of a typical 2-hour CVD devices is extracted to be $\sim 191 \mathrm{~cm}^{2} /(\mathrm{V} . \mathrm{s})$. This value is larger than reported field effect transistor mobilities 40 of RGO, ${ }^{21,36}$ thus substantiating that the ethanol CVD treatment and RGO growth allow 2D charge percolation of the extended $\pi$ electron network.

\section{Conclusions}

In summary, atmospheric pressure ethanol CVD has been 45 demonstrated to reduce oxygen moieties and extend the size of GO. The GO catalysed the deposition of carbon and this growth extension was observed by the SEM and characterized to be RGO using Raman and XPS. Raman spectra indicated that the ethanol grown RGO film possesses lower defect density, better than 50 thermally reduced or hydrazine reduced RGO. We also highlight that our approach reduces electrical resistance and decreases the standard deviation between chips. Our finding suggests that the electrical conductivity is not limited by the presence of defects in the form of carbon vacancies, which cannot be healed by 55 annealing or reduction. To the best of our knowledge, these results constitute the first example of using GO for growth extension. In a broader perspective, the possibility of GO growth represents a big step forward to fabrication of practical and usable electronics.

\section{${ }_{60}$ Notes and references}

${ }^{a}$ School of Material Science and Engineering, Nanyang Technological University, 50 Nanyang Avenue, Singapore, 637553, Singapore; Tel: +656790 4935; E-mail: miytok@ntu.edu.sg

${ }_{65}{ }^{b}$ Institute for Sports Research, Nanyang Technological University, 50 Nanyang Avenue, Singapore 639798

${ }^{c}$ Austrian Institute of Technology (AIT) GmbH, Donau-City Str.1, Vienna, 1220, Austria. Tel: +43-6648251100; E-mail: c.nowak@ait.ac.at

70

${ }^{d}$ Centre for Biomimetic Sensor Science, 50 Nanyang Drive, Singapore, 637553, Singapore.

${ }^{e}$ Loughborough University, Ashby Road, Loughborough, Leicestershire, ${ }_{75}$ LE11 3TU, UK..Tel: +44-1509226311; E-mail:m.a.nimmo@lboro.ac.uk 


\section{Cite this: DOI: 10.1039/c3nr33704a}

pubs.rsc.org/en/content/articlelanding/2013/N ARTICLE TYPE R/c3nr33704a

\section{Acknowledgements}

The authors are grateful for the funding from the Institute for Sports Research (ISR) of Nanyang Technological University (NTU) and the 5 International Graduate School Bio-Nano-Tech; a joint program of University of Natural Resources and Life Sciences Vienna (BOKU), the Austrian Institute of Technology (AIT) and NTU.

$\dagger$ Electronic Supplementary Information (ESI) available: [The ethanol ${ }_{10}$ CVD setup, TEM of CVD treated RGO, graphite 2D Raman spectra, GO synthesis, transfer and reduction methods and details of characterization techniques are described in the document.]. See DOI: 10.1039/b000000x/

\section{References}

15

1. K. S. Novoselov, A. K. Geim, S. V. Morozov, D. Jiang, Y. Zhang, S. V. Dubonos, I. V. Grigorieva and A. A. Firsov, Science, 2004, 306, 666-669.

2. X. Huang, Z. Yin, S. Wu, X. Qi, Q. He, Q. Zhang, Q. Yan, F. Boey and H. Zhang, Small, 2011, 7, 1876-1902.

3. Z. H. Ni, T. Yu, Y. H. Lu, Y. Y. Wang, Y. P. Feng and Z. X. Shen, ACS Nano, 2008, 2, 2301-2305.

4. M. Y. Han, B. Özyilmaz, Y. Zhang and P. Kim, Physical Review Letters, 2007, 98, 206805.

255 T. Ohta, A. Bostwick, T. Seyller, K. Horn and E. Rotenberg, Science, 2006, 313, 951-954.

6. M. Z. Hossain, J. E. Johns, K. H. Bevan, H. J. Karmel, Y. T. Liang, S. Yoshimoto, K. Mukai, T. Koitaya, J. Yoshinobu, M. Kawai, A. M. Lear, L. L. Kesmodel, S. L. Tait and M. C. Hersam, Nat Chem, 2012, 4, 305-309.

7. K. P. Loh, Q. Bao, G. Eda and M. Chhowalla, Nat Chem, 2010, 2, 1015-1024.

8. I. Jung, D. A. Dikin, R. D. Piner and R. S. Ruoff, Nano Letters, 2008, 8, 4283-4287.

35 9. Q. He, H. G. Sudibya, Z. Yin, S. Wu, H. Li, F. Boey, W. Huang, P. Chen and H. Zhang, ACS Nano, 2010, 4, 3201-3208.

10. C.-Y. Su, Y. Xu, W. Zhang, J. Zhao, X. Tang, C.-H. Tsai and L.-J. $\mathrm{Li}$, Chemistry of Materials, 2009, 21, 5674-5680.

11. D. Takagi, Y. Kobayashi and Y. Homma, Journal of the American Chemical Society, 2009, 131, 6922-6923.

12. S. A. Steiner, T. F. Baumann, B. C. Bayer, R. Blume, M. A. Worsley, W. J. MoberlyChan, E. L. Shaw, R. Schlögl, A. J. Hart, S. Hofmann and B. L. Wardle, Journal of the American Chemical Society, 2009, 131, 12144-12154.

45 13. B. Liu, W. Ren, L. Gao, S. Li, S. Pei, C. Liu, C. Jiang and H.-M. Cheng, Journal of the American Chemical Society, 2009, 131, 20822083.

14. H. Bi, S. Sun, F. Huang, X. Xie and M. Jiang, Journal of Materials Chemistry, 2012, 22, 411-416.

50 15. C. Gong, M. Acik, R. M. Abolfath, Y. Chabal and K. Cho, The Journal of Physical Chemistry C, 2012, 116, 9969-9979.

16. C.-Y. Su, Y. Xu, W. Zhang, J. Zhao, A. Liu, X. Tang, C.-H. Tsai, Y. Huang and L.-J. Li, ACS Nano, 2010, 4, 5285-5292.

17. J. H. Hafner, M. J. Bronikowski, B. R. Azamian, P. Nikolaev, A. G.

55 Rinzler, D. T. Colbert, K. A. Smith and R. E. Smalley, Chemical Physics Letters, 1998, 296, 195-202.

18. J. P. Kehrer, Toxicology, 2000, 149, 43-50.

19. C. Berger, Z. Song, T. Li, X. Li, A. Y. Ogbazghi, R. Feng, Z. Dai, A. N. Marchenkov, E. H. Conrad, P. N. First and W. A. de Heer, The Journal of Physical Chemistry B, 2004, 108, 19912-19916.

20. Z. Zeng and K. Natesan, Chemistry of Materials, 2005, 17, 37943801 .
21. M. Larisika, J. Huang, A. Tok, W. Knoll and C. Nowak, Materials Chemistry and Physics, 2012, 15, 304-308.

65 22. J. Li, Y. He, Y. Han, K. Liu, J. Wang, Q. Li, S. Fan and K. Jiang, Nano Letters, 2012, 12, 4095-4101.

23. A. C. Ferrari, J. C. Meyer, V. Scardaci, C. Casiraghi, M. Lazzeri, F. Mauri, S. Piscanec, D. Jiang, K. S. Novoselov, S. Roth and A. K. Geim, Physical Review Letters, 2006, 97, 187401.

70 24. K. N. Kudin, B. Ozbas, H. C. Schniepp, R. K. Prud'homme, I. A. Aksay and R. Car, Nano Letters, 2007, 8, 36-41.

25. D. Yang, A. Velamakanni, G. Bozoklu, S. Park, M. Stoller, R. D. Piner, S. Stankovich, I. Jung, D. A. Field, C. A. Ventrice Jr and R. S. Ruoff, Carbon, 2009, 47, 145-152.

75 26. H. Wang, J. T. Robinson, X. Li and H. Dai, Journal of the American Chemical Society, 2009, 131, 9910-9911.

27. F. Tuinstra and J. L. Koenig, The Journal of Chemical Physics, 1970, 53, 1126-1130.

28. C. Casiraghi, A. Hartschuh, H. Qian, S. Piscanec, C. Georgi, A.

80 Fasoli, K. S. Novoselov, D. M. Basko and A. C. Ferrari, Nano Letters, 2009, 9, 1433-1441.

29. G. Eda, G. Fanchini and M. Chhowalla, Nat Nano, 2008, 3, 270-274.

30. S. Turgeon and R. W. Paynter, Thin Solid Films, 2001, 394, $43-47$.

31. J. C. Lascovich, R. Giorgi and S. Scaglione, Applied Surface Science, 1991, 47, 17-21.

32. Y. Mizokawa, T. Miyasato, S. Nakamura, K. M. Geib and C. W. Wilmsen, Surface Science, 1987, 182, 431-438.

33. K. A. Mkhoyan, A. W. Contryman, J. Silcox, D. A. Stewart, G. Eda, C. Mattevi, S. Miller and M. Chhowalla, Nano Letters, 2009, 9, 1058-1063.

34. Y. Si and E. T. Samulski, Nano Letters, 2008, 8, 1679-1682.

35. X. Dong, D. Fu, W. Fang, Y. Shi, P. Chen and L.-J. Li, Small, 2009, 5, 1422-1426.

36. S. Wang, P.K. Ang, Z. Wang, A.L.L. Tang, J.T.L. Thong, K.P. Loh, $95 \quad$ Nano Letters, 2009, 10, 92-98. 